\title{
Transcatheter closure of atrial septostomy facilitates minimally invasive left ventricular assist device implantation
}

\author{
Wei Quan, BA, ${ }^{\mathrm{a}}$ Aakash Shah, MD, ${ }^{\mathrm{a}}$ Erika Feller, MD, ${ }^{\mathrm{b}}$ Van-Khue Ton, MD, PhD, ${ }^{\mathrm{b}}$ Mark Vesely, MD, \\ Erik Sorensen, $\mathrm{PhD},{ }^{\mathrm{c}}$ Zachary N. Kon, MD, ${ }^{\mathrm{d}}$ and David J. Kaczorowski, MD, ${ }^{\mathrm{a}}$ Baltimore, Md, and \\ New York, NY
}

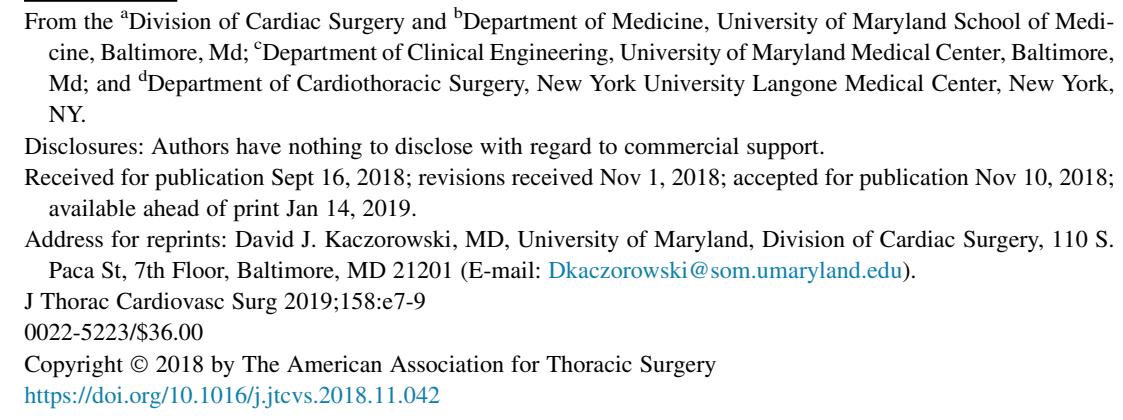

A 41-year-old man with a history of nonischemic cardiomyopathy presented with symptoms of worsening heart failure and was found to have an ejection fraction of $5 \%$ to $10 \%$ with global hypokinesis. He was transferred to our medical center for further management of cardiogenic shock. Despite escalating inotropic support, he continued to decompensate with progressive hypoxemia and worsening end-organ perfusion. He was intubated and peripherally cannulated for venoarterial (VA) extracorporeal membrane oxygenation (ECMO). He developed refractory pulmonary edema (Figure 1, $A$ and $B$ ) while on VA-ECMO and underwent a percutaneous balloon atrial septostomy with a 22-mm $\times 4$-cm Atlas Gold balloon (Bard, Tempe, Ariz). Cardiac catheterization demonstrated an elevated left atrial pressure $(24 \mathrm{~mm} \mathrm{Hg}$ ) before septostomy with a decrease to $10 \mathrm{~mm}$ $\mathrm{Hg}$ postseptostomy. His pulmonary edema subsequently resolved (Figure 1,C) and he was considered an appropriate candidate for left ventricular assist device (LVAD) insertion.

LVAD insertion was performed in a hybrid operating room. A median upper hemisternotomy was performed in an " $\mathrm{L}$ " configuration to the right third intercostal space and the pericardium was opened. A left anterior minithoracotomy incision was created, and the left chest was entered in the fifth intercostal space. At this point, the patient underwent successful atrial septostomy closure with a $15-\mathrm{mm}$ Amplatzer Septal Occluder (Abbott, St Paul, Minn) via the left femoral vein using an 8-French delivery system under fluoroscopic and transesophageal echocardiographic guidance. As venous was being obtained in the left common femoral vein, ECMO flow was briefly turned down to $2 \mathrm{~L} / \mathrm{min}$ and then full flows were resumed.

After the septostomy was closed, VA-ECMO was then converted to full cardiopulmonary bypass. A vent was echocardiography.

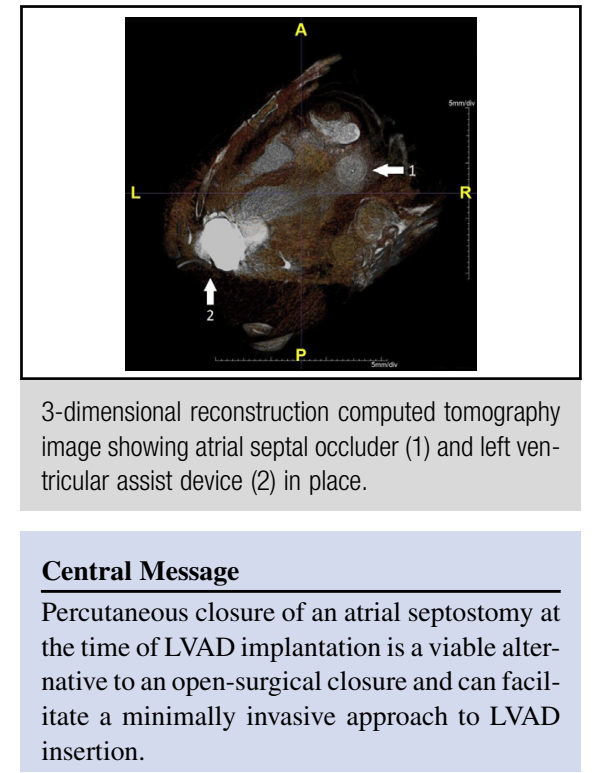

See Commentary on page e11.

inserted into the left ventricle, the apical core was resected, and a HeartWare HVAD (Medtronic, Minneapolis, Minn) was inserted. A partial occlusion clamp was placed on the ascending aorta and an aortotomy was created. The outflow graft of the HeartWare HVAD was positioned appropriately according to length and orientation. It was beveled and then anastomosed to the ascending aorta with a running suture. The clamp was removed and a needle was used to de-air the graft. The patient was weaned off cardiopulmonary bypass while LVAD support was progressively increased without complications. Good right ventricular function, LVAD inflow position, and LV decompression were noted on transesophageal

The patient's postoperative course was uneventful. Transthoracic echocardiography on postoperative day 5 demonstrated appropriate LV unloading and no flow across the atrial septum. Routine computed tomography scan showed appropriate inflow cannula positioning and the septal occluder in place (Figure 2). The patient was discharged on postoperative day 14. Another transthoracic 


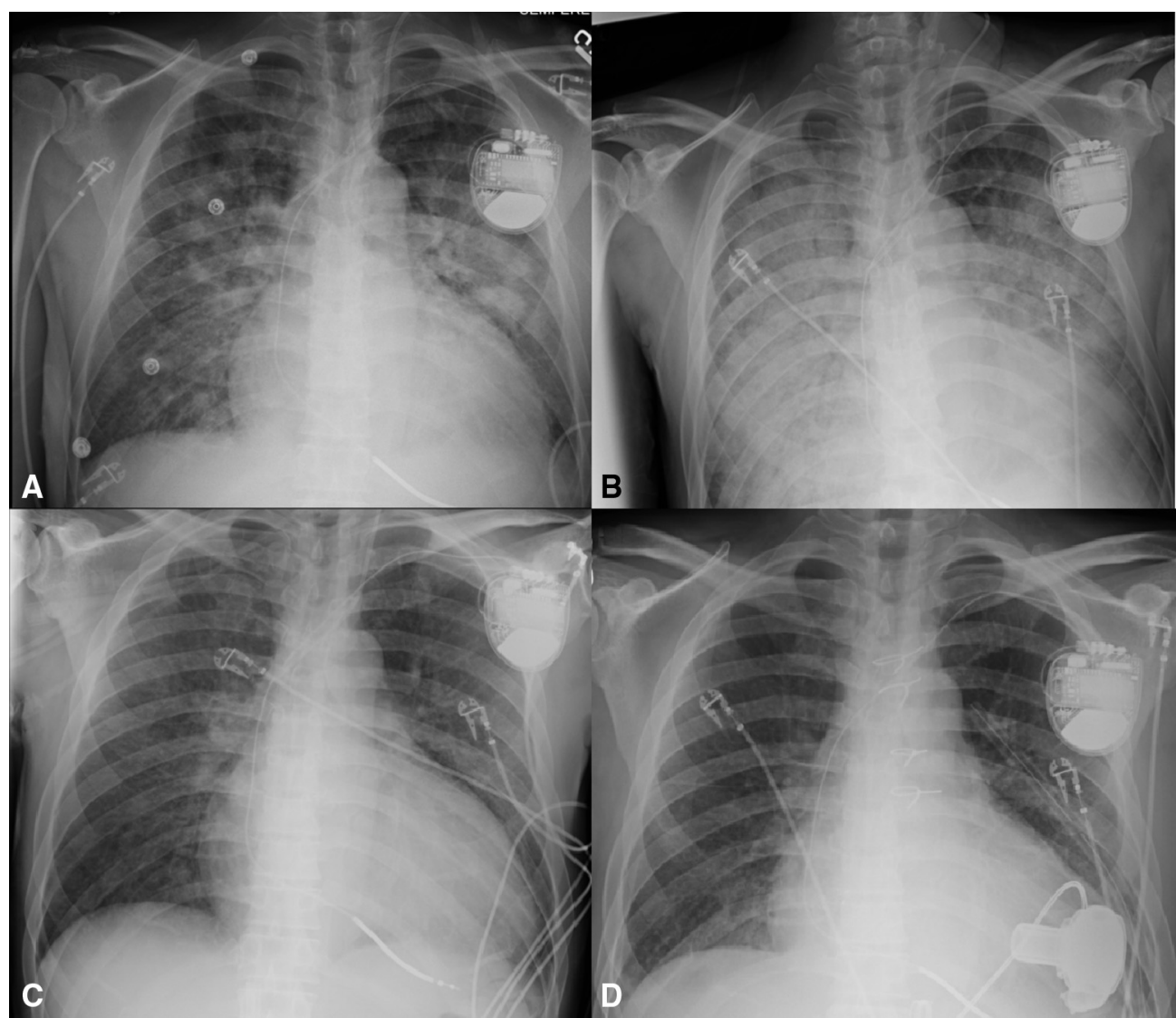

FIGURE 1. Chest radiographs showing (A) mild pulmonary edema in a patient suffering from decompensated congestive heart failure before placement on VA-ECMO, (B) pulmonary edema after placement on VA-ECMO, (C) resolving pulmonary edema after atrial septostomy, and (D) clear lung fields after left ventricular assist device insertion and closure of septostomy.

echocardiography a month from discharge showed no flow across the atrial septum. The patient continues to do well at 6-month follow-up.

\section{DISCUSSION}

Because of its ease of insertion, as well as its ability to provide both hemodynamic support and gas exchange,

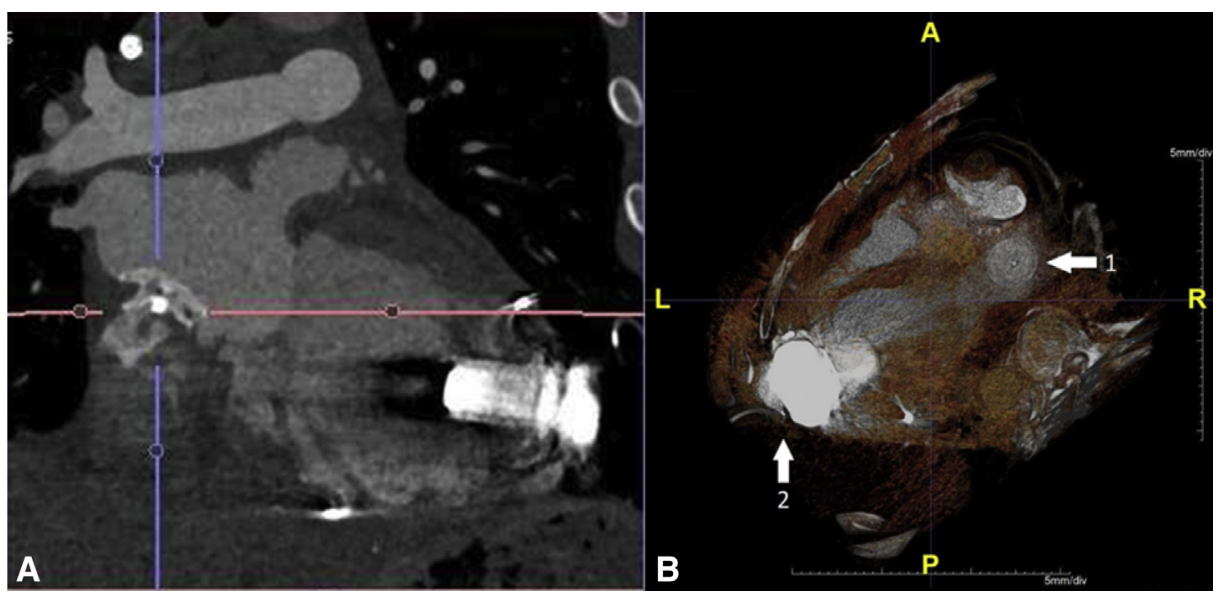

FIGURE 2. (A) Computed tomography image with atrial septal occluder placed at time of minimally invasive LVAD implantation. (B) Three-dimensional reconstruction computed tomography image showing atrial septal occluder (1) and LVAD (2) in place. 
VA-ECMO may be beneficial to patients in cardiogenic shock. ${ }^{1}$ However, LV distension is a recognized complication that can lead to pulmonary edema, increased wall stress, and increased myocardial oxygen consumption. LV decompression with an atrial septostomy can lead to resolution of pulmonary edema. ${ }^{2}$

Patients requiring an LVAD who have had an atrial septostomy require its closure to prevent the development of hypoxemia. ${ }^{3-5}$ These defects can also arise from iatrogenic causes, such as electrophysiology procedures, resulting right to left shunts. ${ }^{6}$ Generally, atrial septal defects are closed surgically at the time of LVAD implantation through a median sternotomy. In other cases, percutaneous closure has been used when they are discovered postoperatively. ${ }^{3-5}$ Although percutaneous closure of septal defects may prevent the need for full sternotomy, one potential disadvantage is the added cost of the closure device.

A minimally invasive approach to LVAD insertion may offer potential benefits, including reduced surgical trauma, less blood loss, less postoperative pain, and better maintenance of right ventricular function. ${ }^{7}$ This approach may also reduce perioperative complications and risks associated with resternotomies. ${ }^{8}$ Further investigation is clearly required confirm these benefits. To our knowledge, this case represents the first description of percutaneous closure of an atrial septostomy at the time of LVAD insertion. Furthermore, the case presented here demonstrates that creation of an atrial septostomy to allow LV decompression during VA ECMO is not prohibitive to a minimally invasive approach to LVAD insertion and that percutaneous transcatheter closure of an atrial septostomy can be safely performed at the time of LVAD insertion to facilitate a minimally invasive approach.

\section{References}

1. Squiers JJ, Lima B, DiMaio JM. Contemporary extracorporeal membrane oxygenation therapy in adults: fundamental principles and systematic review of the evidence. J Thorac Cardiovasc Surg. 2016;152:20-32.

2. Alhussein M, Osten M, Horlick E, Ross H, Fan E, Rao V, et al. Percutaneous left atrial decompression in adults with refractory cardiogenic shock supported with veno-arterial extracorporeal membrane oxygenation. J Card Surg. 2017;32:396-401.

3. Fischer Q, Kirsch M, Brochet E, Juliard JM. Bailout transcatheter closure of patent foramen ovale for refractory hypoxaemia after left ventricular assist device implantation. Interact Cardiovasc Thorac Surg. 2015;21:246-8.

4. Nguyen DQ, Das GS, Grubbs BC, Bolman RM III, Park SJ. Transcatheter closure of patent foramen ovale for hypoxemia during left ventricular assist device support. J Heart Lung Transplant. 1999;18:1021-3.

5. Loforte A, Violini R, Musumeci F. Transcatheter closure of patent foramen ovale for hypoxemia during left ventricular assist device support. J Card Surg. 2012;27: $528-9$.

6. Hong J, Park SJ, Mankad SV, Cetta F Jr, Torres NE, Brown ML. Hypoxemia after an axial flow pump Jarvik-2000 implantation: catheter induced. J Thorac Cardiovasc Surg. 2008; 136:1082-3.

7. Schmitto JD, Molitoris U, Haverich A, Strueber M. Implantation of a centrifugal pump as a left ventricular assist device through a novel, minimized approach: upper hemisternotomy combined with anterolateral thoracotomy. J Thorac Cardiovasc Surg. 2012;143:511-3.

8. Reichart D, Brand CF, Bernhardt AM, Schmidt S, Schaefer A, Blankenberg S, et al. Analysis of minimally invasive left thoracotomy HVAD implantation-a single-center experience. Thorac Cardiovasc Surg. 2019;67:170-5. 